\title{
Conditions for Low Voltage Microanalysis and X-ray Mapping
}

\author{
Richard Wuhrer ${ }^{1}$ and Ken Moran ${ }^{2}$ \\ ${ }^{1}$ Western Sydney University, Advanced Materials Characterisation Facility (AMCF), Australia. \\ ${ }^{2}$ Moran Scientific Pty Ltd, 4850 Oallen Ford Road, Bungonia, NSW, Australia.
}

Traditional microanalysis using energy dispersive X-ray spectroscopy (EDS) involves using higher accelerating voltages in the range of $15 \mathrm{kV}$ to $25 \mathrm{kV}$ in order to excite the well-known K-Lines in the atomic spectra. However, the use of higher accelerating voltages usually results in larger interaction volumes [1], which consequently degrades the spatial resolution of the X-ray image and increases the analytical volume.

With the development and advancement of large area silicon drift detectors (SDD's), the sensitivity for X-rays in the low energy part of the spectrum has substantially improved. This, and high count rate throughput, is now allowing the possibility of operating the SEM at much lower accelerating voltages and subsequently reducing the interaction volume of the electron beam with the material as well as achieving higher spatial resolution information. Furthermore, SDD's can now be purchased with varying windows [2] and also windowless systems [3-6] allowing greater sensitivity of the lower energy X-rays. Figure 1 shows the EDS spectrum from an Amptek detector with two different window materials $(\mathrm{C} 1$ and $\mathrm{C} 2$ ). These windows are made from $90 \mathrm{~nm}$ and $40 \mathrm{~nm}$ silicon nitride (Si3N4) with a very thin aluminium coating to extend the low energy response [2]. The $\mathrm{C} 2$ window has excellent transmission for low $\mathrm{Z}$ elements with ten times more carbon X-rays.

By lowering the accelerating voltage, this forces the selection of X-ray lines with low excitation energy such as $\mathrm{L}$ and $\mathrm{M}$ family lines between $0-4 \mathrm{kV}$, rather than using $\mathrm{K}$ and $\mathrm{L}$ lines between $0-20 \mathrm{kV}[1,3,6]$. The measurement of low energy L line X-rays is complicated by low fluorescence yield, an increase in X-ray absorption, numerous X-ray interferences from other elements within the sample, and less accurately determined mass absorption coefficients [1, 3-6]. The overlaps of K-line X-rays from light elements with L and M line X-rays from heavier elements limit the low voltage analysis and mapping capabilities of conventional microanalysis systems [7].

The use of transition element L-lines generally results in poor accuracy as well as poor sensitivity [811]. Furthermore, operating at low voltages has limitations due to the large quantification errors existing on the lower energy X-ray lines [11]. As can be seen in Figure 1c, the EDS spectra shows the hard facing material of $\mathrm{NbC}$ particles in white cast iron, has numerous L lines overlapping each other. The subsequent generation of X-ray maps collected at $8 \mathrm{kV}$ using $\mathrm{K}$ lines (Figure $2 \mathrm{~b}-\mathrm{e}$ ) and L lines (Figure 2f, h-j) shows major errors for Mn L line x-ray. Furthermore, the quantification results using the L lines are totally incorrect. This can be minimized by more precise calibration of the detector parameters (possibly due to non-linearities in the detector electronics) to enable better correction of the overlaps in the low energy range.

Extensive work is still required on fundamental parameters [8], algorithms and mass absorption coefficients before any accurate quantitative analysis can be performed [9-11], especially with the low voltage regime of multi-element materials. These multi-element materials that have many overlaps, are only good for qualitative analysis. 
High quality quantitative analysis at low voltage is best carried out using a standards based method [8]. Standardless analysis has many more parameters to be determined for accurate analysis compared to standards based analysis [8], however there are still many sources of errors. The SDDs have to be calibrated more carefully and using different calibration standards than that used for conventional standards at higher accelerating voltages.

References:

[1] D. C. Bell and N. Erdman, "Low Voltage Electron Microscopy", Wiley, 2013.

[2] http://www.amptek.com/products/c-series-low-energy-x-ray-windows/

[3] M. Meisnara, S. Lozano-Pereza, M. Moodya and J. Holland, Micron 66, 16-22, 2014.

[4] R. Wuhrer, L. Guja, D. Merritt and K. Moran, Microsc. Microanal. 20 (Suppl 3), 634-635, 2014.

[5] S. Burgess, X. Li,, J. Holland, Microsc. Anal. 27, 8, 2013.

[6] L. Moran, K. Moran and R. Wuhrer, Microsc. Microanal. 20 (Suppl 3), p678-679, 2014.

[7] J. J. Friel, C. E. Lyman, Microsc. Microanal. 12, 2-15, 2006.

[8] R. Gauvin, Microscopy and Microanalysis, 18, 915-940, 2012.

[9] P. K. Carpenter, AMAS XIII-The 13 ${ }^{\text {th }}$ Biennial AMAS Symposium, Hobart, 132-133, Feb, 2015.

[10] J. Donovan, P. Pinard, and S. Richter, AMAS XIII Symposium, Hobart, 122-123, Feb, 2015

[11] P. Pinard and S. Richter, AMAS XIII-The $13^{\text {th }}$ Biennial Australian Microbeam Analysis Symposium, Hobart, 124-125, Feb, 2015.

[12] R. Wuhrer and K. Moran, EMAS Proceedings 2015, Slovenia, Nov 2015.
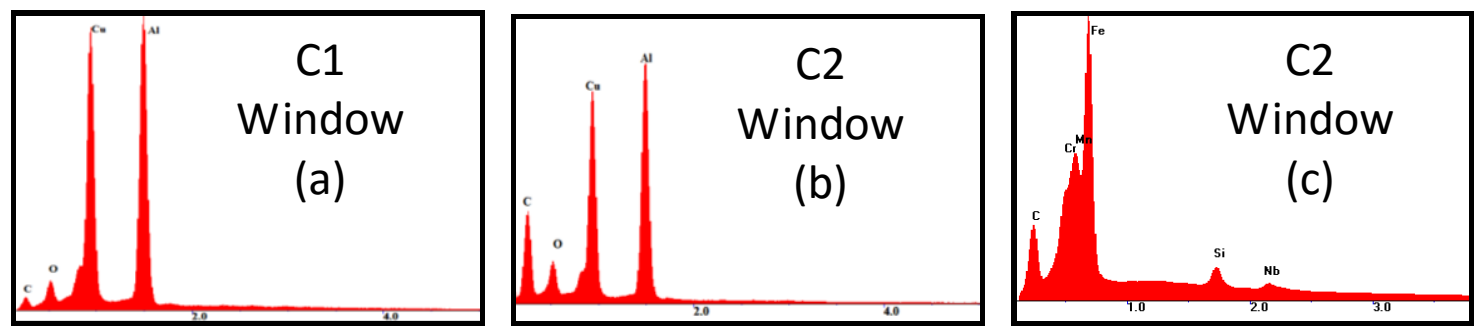

Figure 1. EDS spectra from an Amptek Fast123 detector, collected at $E_{0}=8 \mathrm{kV}$. a) $90 \mathrm{~nm}(\mathrm{C} 1)$ window of a contaminated $\mathrm{Cu}-\mathrm{Al}$ grid, b) $40 \mathrm{~nm}$ (C2) window of a contaminated $\mathrm{Cu}-\mathrm{Al}$ grid (same area) and c) Spectra (from C2 window detector) of a white cast iron (WCI) hard-facing material that has $\mathrm{NbC}$ particles present Reproduced from [12].

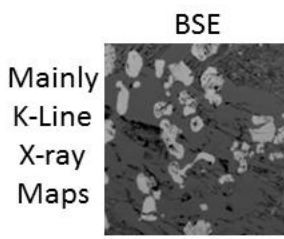

a.

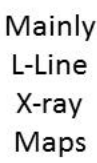

Mainly

L-Line

Maps

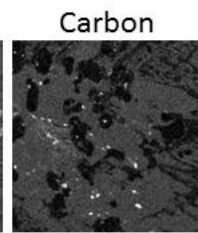

b.

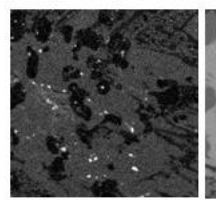

g.

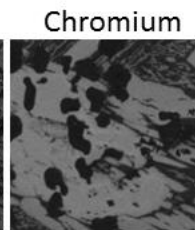

c.

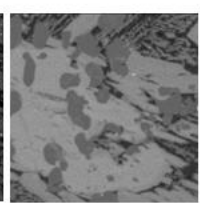

h.

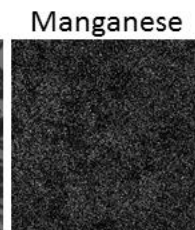

d.

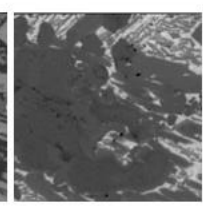

i.

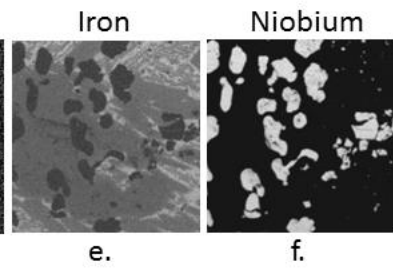

e.

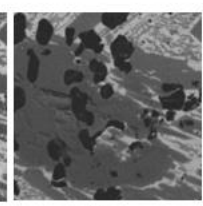

j.

Figure 2. Corrected $\mathrm{x}$-ray maps taken at $8 \mathrm{kV}$ of hard-facing material with $\mathrm{NbC}$ particles in white cast iron. a) BSE image, b) Carbon K-Line, c) Chromium K-line, d) Mn K-line, e) Fe K-line, f) Nb L-line, g) Carbon K-Line, h) Chromium L-line, i) Mn L-line and j) Fe L-line. Notice the Mn X-ray map for the Kline and L-line X-ray maps are totally different, due to the linearity and overlap problems occurring at the L-lines for Cr, Mn, and Fe. Reproduced from [12]. 